\title{
Alterações bioquímicas associadas a injúrias foliares visíveis em plantas jovens de Psidium guajava 'Paluma' mantidas em ambiente contaminado por ozônio
}

\author{
Ana Paula de Souza Dias ${ }^{1}$, Mirian Cilene Spasiani Rinaldi ${ }^{1}$ e Regina Maria de Moraes ${ }^{1,2}$
}

Recebido: 07.07.2006; aceito: 24.05.2007

\begin{abstract}
Biochemical alterations associate with visible foliar injury in saplings of Psidium guajava 'Paluma' maintained in an environment polluted by ozone). Psidium guajava 'Paluma' is sensitive to ozone, to which reacts with characteristic visible foliar injuries. The concentration of ascorbic acid (AA) and the activity of enzymes superoxide dismutase (SOD) and peroxidase (POD) had been evaluated in new and old leaves of plants exposed $(n=13)$ to ozone in a contaminated area (Ibirapuera, São Paulo, SP) and in a controlled environment (glasshouse with filtered air) to assess the occurrence of latent injuries (alteration in AA, SOD and/or POD) and if the occurrence of these differed between leaves with or without visible injuries. The AA concentration was higher in plants of Ibirapuera, without difference between new or old and with or without visible injuries. The activity of SOD and POD differed between new and old leaves, but not with relation to the site of exposition and the presence or absence of foliar injuries. There was no significant decrease in biomass in plants exposed in Ibirapuera. We conclude that in P. guajava 'Paluma' SOD and POD are not related to the protection against visible foliar injuries and that the AA is a indicator of latent injury.
\end{abstract}

Key words: ascorbic acid, foliar injury, peroxidase, superoxide dismutase

RESUMO - (Alterações bioquímicas associadas a injúrias foliares visíveis em plantas jovens de Psidium guajava 'Paluma' mantidas em ambiente contaminado por ozônio). Psidium guajava 'Paluma' é sensível ao ozônio, ao qual responde com características injúrias foliares visíveis. Foram avaliadas a concentração de ácido ascórbico (AA) e a atividade das enzimas superóxido dismutase (SOD) e peroxidases (POD) em folhas novas e velhas de plantas expostas $(\mathrm{n}=13)$ em região contaminada por ozônio (Ibirapuera, São Paulo, SP) e em um ambiente controlado (casa de vegetação com ar filtrado), com o objetivo de avaliar a ocorrência de injúrias latentes (alteração em AA, SOD e/ou POD) e se a ocorrência destas diferia entre folhas com ou sem injúrias visíveis. A concentração de AA foi maior nas plantas do Ibirapuera, sem diferença entre novas ou velhas e com ou sem injúrias. A atividade de SOD e POD diferiu entre folhas novas e velhas, mas não com relação ao local de exposição e a presença ou ausência de injúrias foliares. Não houve decréscimo na biomassa das plantas expostas no Ibirapuera. Conclui-se que em P. guajava 'Paluma' SOD e POD não estão relacionadas à proteção contra injúrias foliares visíveis nessa espécie e que o AA é um indicador de injúria latente.

Palavras-chave: ácido ascórbico, injúrias foliares, peroxidases, superóxido dismutase

\section{Introdução}

O ozônio $\left(\mathrm{O}_{3}\right)$ presente na troposfera é, atualmente, o poluente que mais causa danos à vegetação e perdas na produtividade agrícola em escala mundial (Krupa et al. 2001). Ele é formado na atmosfera através de reações fotoquímicas entre óxidos de nitrogênio e compostos orgânicos voláteis, os quais são provenientes principalmente da queima de combustíveis fósseis. É o poluente aéreo com maior número de ultrapassagens dos padrões legais de qualidade do ar na cidade de São Paulo (CETESB 2006).

Em algumas espécies sensíveis, o $\mathrm{O}_{3}$ induz a manifestação de injúrias foliares visíveis, que podem ser cloroses, necroses ou pontuações vermelhas ou marrons na superfície adaxial do limbo, entre as nervuras das folhas mais velhas (ICP-forests 2006, Sanz et al. 2002). Embora todos esses sintomas sejam considerados injúrias visíveis e sejam usados na identificação das espécies bioindicadoras sensíveis (De Temmerman et al. 2004), eles diferem quanto à sua

1. Instituto de Botânica, Caixa Postal 3005, 01061-970 São Paulo, SP, Brasil

2. Autor para correspondência: regmorae@yahoo.com.br 
gravidade, com os mais deletérios provocando morte celular, por exemplo, necroses em Nicotiana tabacum Bel W3 (Klumpp et al. 2006) e os menos prejudiciais correspondendo a manchas causadas pelo acúmulo de substâncias fenólicas nos vacúolos, como é verificado em Psidium guajava 'Paluma' (Alves 2006).

Em muitas espécies, antes que ocorra a manifestação de sintomas visíveis, as plantas expostas a poluentes apresentam alterações fisiológicas ou bioquímicas que são chamadas de injúrias latentes (Dassler \& Bortitz 1988). Uma das primeiras injúrias latentes induzidas pelo $\mathrm{O}_{3}$ é a alteração da concentração e da atividade dos constituintes do sistema de defesa antioxidante em decorrência da formação de espécies ativas de oxigênio (EAO). As EAO são capazes de causar danos aos constituintes celulares, como membranas e ácidos nucléicos. Entretanto, elas são uma decorrência normal do metabolismo do oxigênio, de modo que, na célula viva há um delicado balanço entre a produção de EAO e a atividade do sistema oxidante, que as neutraliza. Fatores de estresse, como a poluição aérea, podem alterar esse balanço finamente regulado levando ao acúmulo de EAO e dando origem ao estresse oxidativo (Bray et al. 2000).

O sistema de defesa antioxidante é formado por enzimas (como as peroxidases e a superóxido dismutase) e compostos não enzimáticos, como o ácido ascórbico, entre outros. O ácido ascórbico atua no reparo de ácidos graxos insaturados, além de ser capaz de seqüestrar radicais livres, impedindo-os de reagir com componentes celulares vulneráveis (Chameides 1989). O ácido ascórbico localizado no apoplasto constitui a primeira linha de defesa da planta contra as EAO (Chameides 1989). A enzima superóxido dismutase catalisa a dismutação do radical superóxido em peróxido de hidrogênio e oxigênio. $\mathrm{O}$ peróxido de hidrogênio assim formado é, a seguir, reduzido a água pelas peroxidases na presença de ácido ascórbico (Bowler et al. 1992). Alguns estudos verificaram que plantas que possuem maior atividade do sistema antioxidante são mais resistentes ao ozônio e manifestam menos sintomas foliares visíveis (Iqbal et al. 1996, Gadallah 2000, Burkey et al. 2006).

Psidium guajava 'Paluma' é sensível ao $\mathrm{O}_{3}$ desenvolvendo injúrias foliares visíveis quando exposta a este poluente (Furlan et al. 2007, Pina \& Moraes 2007). Com base nisso, decidiu-se avaliar a atividade do sistema antioxidante em folhas de plantas expostas ao $\mathrm{O}_{3}$ com e sem manifestação de injúrias e também num grupo referência mantido sob ar filtrado, com o objetivo de verificar se a manifestação de injúrias foliares visíveis induzidas pelo $\mathrm{O}_{3}$ é precedida por alterações na concentração de ácido ascórbico (AA) e na atividade das enzimas superóxido dismutase (SOD) e peroxidases (POD) e se as folhas com injúrias foliares apresentavam menor concentração de ácido ascórbico e menor atividade das enzimas superóxido dismutase e peroxidases do que as folhas sem injúrias.

Embora várias espécies vegetais sejam rotineiramente empregadas como bioindicadores da poluição por ozônio em países das regiões temperadas (Filella et al. 2005, Klumpp et al. 2006), pouco se sabe sobre a sensibilidade de espécies tropicais a este poluente. A seleção de espécies nativas com potencial bioindicador para utilização em programas de biomonitoramento nas regiões tropicais é de grande importância, pois estas espécies estão adaptadas às condições ambientais dessas regiões e produzirão respostas com maior significado ecológico (Manning 2003). Nesse sentido, este estudo buscou a obtenção de informações que complementassem o conhecimento existente sobre a sensibilidade de P. guajava 'Paluma' ao ozônio, visando sua proposição como espécie tropical bioindicadora desse poluente.

\section{Material e métodos}

Área de estudo - O Parque Municipal do Ibirapuera, zona sul de São Paulo, SP (2334'S e 46³9'W, 750 $\mathrm{m}$ sobre o nível do mar) constituiu a área de estudo. É a região que registra as mais altas concentrações de $\mathrm{O}_{3}$ na cidade de São Paulo. As concentrações dos demais poluentes, no entanto, são negligenciáveis (CETESB 2006). Dados horários de concentração de $\mathrm{O}_{3}$ e das condições climáticas (temperatura e umidade relativa) foram obtidos em www.cetesb.sp.gov.br/Ar/ ar_dados_horarios.asp. Foram calculadas as concentrações médias diárias e do período e identificadas as máximas horárias. Calculou-se também a exposição acumulada a concentrações horárias de $\mathrm{O}_{3}$ superiores a $40 \mathrm{ppb}$ durante o período em que a radiação global foi maior ou igual a $50 \mathrm{~W} \mathrm{~m}^{-2}$, AOT40 (do inglês: Accumulated Ozone Threshold above $40 \mathrm{ppb}$ h) índice proposto na Europa para proteção de sua vegetação (Fuhrer et al. 1997) e usado aqui apenas em termos comparativos, pois não existe um índice semelhante para regiões tropicais. A área 
de referência foi uma casa de vegetação com ar filtrado, pertencente ao Instituto de Botânica. Ela possui um sistema de filtros de carvão ativado para retirada de gases e filtros de lã de vidro e papel, para retirada de partículas. Possui sensores de temperatura e um termohigrógrafo. O monitoramento da concentração de $\mathrm{O}_{3}, \mathrm{NO}_{2}$ e $\mathrm{SO}_{2}$ no interior da casa de vegetação foi feito por meio de medidas descontínuas realizadas com um equipamento Tri-Gás (Energética, RJ, BR), enquanto a de Material Particulado (MP) foi determinada com um Amostrador de Grandes Volumes (AGV 10, Energética, RJ, Brasil).

Cultivo e exposição das plantas - Adotou-se o modelo proposto por Arndt \& Schweizer (1991) com pequenas adaptações. Plantas de mesma procedência, com cerca de $30 \mathrm{~cm}$ de altura, obtidas de um produtor rural especializado em produção de mudas por estaquia, foram plantadas em vasos de 1,5 L utilizando como substrato casca de coco em pó. A seguir, os vasos permaneceram por 30 dias em casa de vegetação para que as plantas se recuperassem do transplante. As plantas foram irrigadas por capilaridade, através de cordas de náilon inseridas na base dos vasos que faziam contato com as raízes e com reservatórios de água. Elas receberam quinzenalmente $100 \mathrm{ml}$ de adubo comercial Peters $^{\circledR}$ (N:P:K 10:30:20). Durante o período de 3 de março a 4 de maio de 2006, 13 vasos com plantas de $P$. guajava 'Paluma' foram expostos em cada área.

Injúrias foliares visíveis - Foram realizadas visitas semanais ao Ibirapuera para avaliação de injúrias foliares visíveis e também para os cuidados com as plantas. Em P. guajava 'Paluma' os sintomas foliares manifestam-se como pequenas pontuações vermelhas intervenais na superfície adaxial, surgindo inicialmente nas folhas mais velhas (Furlan et al. 2007, Pina \& Moraes 2007). Os critérios utilizados para classificar os danos como sendo causados pelo $\mathrm{O}_{3}$ seguem a literatura de referência (ICP-forests 2006, Novak et al. 2003).

Foram avaliadas a incidência e a severidade dos sintomas, segundo Chappelka et al. (1997): incidência é o número de indivíduos com sintomas foliares em porcentagem do número total de indivíduos e severidade é o número de folhas com danos em porcentagem do número total de folhas das plantas que possuem danos.

Uma outra medida de severidade empregada foi a estimativa da porcentagem da área foliar ocupada por danos. Os danos foliares foram classificados em cinco níveis de injúrias: nível 1 , folhas com 1-5\% da superfície do limbo com injúrias; nível 2, folhas com $6-25 \%$ de injúrias; nível 3 , folhas com $26-50 \%$ de injúrias; nível 4, folhas com 51-75\% de injúrias e nível 5, folhas com $76-100 \%$ de injúrias. Posteriormente, as cinco classes de porcentagem foram utilizadas para calcular o Índice de Injúria foliar (IIF), segundo Furlan et al. (2007):

$$
\operatorname{IIF}(\%)=\frac{\left(\mathrm{N}_{1} \times 1\right)+\left(\mathrm{N}_{2} \times 2\right)+\left(\mathrm{N}_{3} \times 3\right)+\left(\mathrm{N}_{4} \times 4\right)+\left(\mathrm{N}_{5} \times 5\right)}{\left(\mathrm{N}_{0}+\mathrm{N}_{1}+\mathrm{N}_{2}+\mathrm{N}_{3}+\mathrm{N}_{4}+\mathrm{N}_{5}\right) \times 5} \times 100
$$

onde $\mathrm{N}_{1}, \mathrm{~N}_{2}, \mathrm{~N}_{3}, \mathrm{~N}_{4}, \mathrm{~N}_{5}$ e $\mathrm{N}_{0}$ eram respectivamente os números de folhas que se enquadravam nos níveis $1,2,3,4,5$ e com nenhuma injúria.

Antioxidantes - Ao final da exposição, foram retiradas duas amostras de folhas frescas de cada planta com danos e sem danos $(n=6)$, uma do terceiro nó mais velho e outra do terceiro nó mais novo, para análise dos três antioxidantes.

Ácido ascórbico (AA) - folhas frescas $(0,500 \mathrm{~g})$ foram homogeneizadas em $12 \mathrm{~mL}$ de solução aquosa contendo EDTA-Na $2(0,07 \%)$ e ácido oxálico $(0,5 \%)$ e centrifugadas $(18.000 \mathrm{rpm})$ a $2{ }^{\circ} \mathrm{C}$ durante 30 minutos. Após isso, adicionou-se $2,5 \mathrm{~mL}$ de diclorofenol-indofenol ao extrato resultante e realizou-se uma primeira leitura em espectrofotômetro $(=520 \mathrm{~nm})$, então, adicionou-se $0,05 \mathrm{~mL}$ de ácido ascórbico (1\%) a essa solução e fez-se uma segunda leitura. A concentração de ácido ascórbico foi estimada a partir da diferença dessas duas leituras (Keller \& Schweizer 1977).

Superóxido dismutase (SOD) - A atividade da enzima foi determinada segundo o método de Osswald et al. (1992), medindo-se sua capacidade de inibir a redução fotoquímica do azul de nitro tetrazólio (NBT). O extrato enzimático foi obtido através da homogeneização e centrifugação $(10.000 \mathrm{rpm})$ das folhas frescas $(0,350 \mathrm{~g})$ com polivinilpolipirrolidona (PVPP) 4\% e tampão fosfato $(50 \mathrm{mM}, \mathrm{pH}=7,5)$ contendo EDTA $1 \mathrm{mM}, \mathrm{NaCl} 50 \mathrm{mM}$ e ácido ascórbico $1 \mathrm{mM}$. A seguir, foram adicionados ao extrato resultante, $0,5 \mathrm{~mL}$ de metionina $(0,13 \mathrm{mM})$, $0,5 \mathrm{~mL}$ de EDTA $(0,54 \mathrm{mM}), 0,8 \mathrm{~mL}$ de tampão fosfato de potássio $(0,1 \mathrm{M}, \mathrm{pH} 7,0), 0,2 \mathrm{~mL}$ de riboflavina ( $1 \mathrm{mM})$ e $0,5 \mathrm{~mL}$ de NBT $(0,44 \mathrm{mM})$. Os tubos contendo a solução obtida foram expostos a luz fluorescente $(80 \mathrm{~W})$ por 30 minutos. Extratos 
preparados seguindo o mesmo procedimento foram mantidos no escuro. A absorbância da solução foi medida em espectrofotômetro $(=560 \mathrm{~nm})$ em ambos os tipos de extrato (iluminado e não iluminado) e a diferença entre as duas absorbâncias foi considerada para a determinação da atividade da SOD, que constituiu na inibição da redução do NBT, pela dismutação enzimática do superóxido. Neste método, a riboflavina reduzida fotoquimicamente gera $\mathrm{O}_{2}{ }_{2}^{-}, \mathrm{O}$ qual reduz o NBT. Na presença de SOD esta redução é inibida. Os resultados são expressos em unidades de enzima, que é a quantidade de SOD necessária para reduzir a taxa de redução do NBT em $50 \%$.

Peroxidase (POD) - Folhas frescas $(0,300 \mathrm{~g})$ foram homogeneizadas com tampão fosfato $(0,1 \mathrm{M}, \mathrm{pH} 7,0)$ e PVPP 4\% e em seguida foram centrifugadas (18.000 rpm). A seguir, adicionou-se ao extrato resultante tampão fosfato $(0,1 \mathrm{M}, \mathrm{pH} 5,0)$, fenilendiamina ( $1 \%)$ e peróxido de hidrogênio $(0,3 \%)$ e determinou-se a absorbância em espectrofotômetro $(=485 \mathrm{~nm})$, como descrito por Klumpp et al. (1989). O cromóforo formado é o fenilendiamina na forma oxidada. A absorbância do complexo $\mathrm{H}_{2} \mathrm{O}_{2}$-POD formada foi medida de 30 em 30 segundos até alcançar 600 segundos, indicando a atividade da POD durante a redução do peróxido de hidrogênio.

Crescimento - No final da exposição, folhas, caules e raízes de todas as plantas foram coletadas e secas separadamente em estufa a $70^{\circ} \mathrm{C}$ para o determinação da massa seca em balança semi-analítica.

Estatística - As concentrações médias de ácido ascórbico, atividade de SOD e POD em folhas novas e velhas, com e sem injúrias e os dados de massa seca foram comparados através de análise de variância, sendo as diferenças discriminadas através de teste de comparações múltiplas de StudentNeumman-Keuls $(\mathrm{p}<0,05)$.

\section{Resultados}

Durante o período de estudo, foram registrados na área poluída temperatura média de $21^{\circ} \mathrm{C}$ e umidade relativa de $82 \%$. Na casa de vegetação a temperatura média ficou em $23{ }^{\circ} \mathrm{C}$ e a umidade relativa em $74 \%$. As condições climáticas durante o período de exposição não foram favoráveis à formação de $\mathrm{O}_{3}$, sendo registradas no Ibirapuera concentrações mais baixas do que a média dos últimos cinco anos para o mesmo período (CETESB 2006). A concentração média de $\mathrm{O}_{3}$ no período foi de $28 \mu \mathrm{g} \mathrm{m}^{-3}$ e máxima horária, $182 \mu \mathrm{g} \mathrm{m}^{-3}$. Na área de referência, a concentração horária de $\mathrm{O}_{3}$ foi sempre inferior a $10 \mu \mathrm{g} \mathrm{m}^{-3}$, registrando média de $3 \mu \mathrm{g} \mathrm{m}^{-3}$. As concentrações dos demais poluentes foram negligenciáveis (tabela 1).

$\mathrm{O}$ início da manifestação de injúrias foliares visíveis ocorreu depois de 24 dias de permanência das plantas na área poluída (tabela 2). As plantas mantidas na área de referência não apresentaram injúrias foliares induzidas pelo $\mathrm{O}_{3}$ em nenhum momento. Ao final da exposição, a incidência de injúrias foi de $31 \%$ das plantas. A severidade e o IIF foram baixos e atingiram seus valores mais altos aos 24 dias de exposição.

Não houve diferenças nas concentrações de AA de folhas de plantas com e sem injúrias, novas ou velhas (figura 1), mas houve entre folhas de plantas mantidas na área poluída e no ar filtrado $(\mathrm{p}<0,05)$.

A atividade das enzimas SOD e POD apresentou respostas semelhantes, com diferenças significativas entre folhas novas e folhas velhas, sendo que as mais velhas apresentavam atividades mais altas (figura 1). Porém, na comparação entre plantas mantidas sob ar filtrado e plantas expostas ao $\mathrm{O}_{3}$, assim como na comparação entre plantas que apresentavam injúrias e aquelas que não as apresentavam, não foram verificadas diferenças significativas (figura 1). Também não houve diferença significativa entre as

Tabela 1. Médias de temperatura $\left({ }^{\circ} \mathrm{C}\right)$, umidade relativa e concentrações de poluentes: ozônio $\left(\mathrm{O}_{3}\right)$, dióxido de enxofre $\left(\mathrm{SO}_{2}\right)$, dióxido de nitrogênio $\left(\mathrm{NO}_{2}, \mu \mathrm{g} \mathrm{m}^{-3}\right)$ e material particulado (MP, ppb) durante o período de estudo, máxima horária de $\mathrm{O}_{3}$ e AOT40 (concentração horária acumulada de $\mathrm{O}_{3}$ acima de $40 \mathrm{ppb}$ ).

\begin{tabular}{|c|c|c|c|c|c|c|c|c|}
\hline & $\begin{array}{c}\mathrm{T} \\
\left({ }^{\circ} \mathrm{C}\right)\end{array}$ & $\begin{array}{l}\text { UR } \\
(\%)\end{array}$ & $\begin{array}{l}\mathrm{O}_{3} \text { média } \\
\left(\mu \mathrm{g} \mathrm{m}^{-3}\right)\end{array}$ & $\begin{array}{c}\mathrm{O}_{3} \text { máx. } \\
\text { horária }\left(\mu \mathrm{g} \mathrm{m}^{-3}\right)\end{array}$ & $\begin{array}{l}\text { AOT40 } \\
(\mathrm{ppb})\end{array}$ & $\begin{array}{c}\mathrm{SO}_{2} \\
\left(\mu \mathrm{g} \mathrm{m}^{-3}\right)\end{array}$ & $\begin{array}{c}\mathrm{NO}_{2} \\
\left(\mu \mathrm{g} \mathrm{m}^{-3}\right)\end{array}$ & $\begin{array}{l}\text { MP } \\
(\mathrm{ppb})\end{array}$ \\
\hline Ibirapuera & 21 & 82 & 28 & 182 & 1557 & 5 & 32 & 27 \\
\hline Casa de Vegetação & 23 & 74 & 3 & 10 & 0 & nd & 6 & 7 \\
\hline
\end{tabular}

nd - não detectável 
Tabela 2. Progressão de injúrias foliares visíveis em plantas de P. guajava 'Paluma' expostas no Parque Municipal do Ibirapuera. Incidência, severidade e índice de injúria foliar (IIF, todos em \%) e AOT40 (concentração horária acumulada de $\mathrm{O}_{3}$ acima de 40 ppb).

\begin{tabular}{|c|c|c|c|c|c|}
\hline Data & Dias de exposição & Incidência & Severidade & IIF & AOT40 \\
\hline 03/03/06 & 0 & 0 & 0 & 0 & 0 \\
\hline 06/03/06 & 3 & 0 & 0 & 0 & 0,5 \\
\hline $13 / 03 / 06$ & 10 & 0 & 0 & 0 & 250 \\
\hline $20 / 03 / 06$ & 17 & 0 & 0 & 0 & 470 \\
\hline $27 / 03 / 06$ & 24 & 6 & 14,0 & 2,8 & 480 \\
\hline 03/04/06 & 31 & 12 & 11,4 & 2,3 & 480 \\
\hline $10 / 04 / 06$ & 38 & 12 & 11,4 & 2,3 & 631 \\
\hline $17 / 04 / 06$ & 45 & 18 & 9,0 & 1,8 & 1085 \\
\hline $24 / 04 / 06$ & 52 & 25 & 9,8 & 1,9 & 1236 \\
\hline $04 / 05 / 06$ & 60 & 31 & 9,3 & 1,8 & 1557 \\
\hline
\end{tabular}

massas secas de folhas, caules e raízes entre as plantas que permaneceram no Ibirapuera ou sob ar filtrado (tabela 3).

\section{Discussão}

As injúrias foliares visíveis presentes em plantas jovens de $P$. guajava 'Paluma' mantidas no Parque Ibirapuera podem ser consideradas como induzidas pelo ozônio porque obedecem a todos os critérios descritos na literatura de referência: começam nas folhas mais velhas; não cobrem nervuras; afetam apenas a superfície adaxial do limbo foliar (Skelly et al. 1999, Sanz et al. 2002, Novak et al. 2003, ICP Forest 2006). As pontuações intervenais na superfície adaxial do limbo das folhas mais velhas são consideradas o mais típico sintoma de injúria induzida pelo $\mathrm{O}_{3}$ em espécies latifoliadas (ICP-forests 2006). Além disso, foram muito semelhantes às obtidas por Furlan et al. (2007) em experimento realizado com a mesma cultivar em condições semi-controladas (fumigação com ozônio em câmaras de topo aberto) e por Pina \& Moraes (2007), em condições de campo.

A ocorrência dessas injúrias indica que as concentrações de $\mathrm{O}_{3}$ verificadas no Ibirapuera, apesar de não terem sido altas, foram fitotóxicas. As oscilações nos valores de severidade e IIF devem-se à abscisão de folhas com injúrias e à manifestação de danos em folhas que ainda não os apresentavam. $\mathrm{O}$ $\mathrm{O}_{3}$ induz ao estresse oxidativo, antecipando a senescência foliar (Pell et al. 1994) e reduzindo o tempo de vida da folha.

Furlan et al. (2007) verificaram correlação entre a AOT40 e o IIF maior do que a obtida no presente estudo. Isso se deve às diferenças entre as condições de exposição nos dois experimentos. Em Furlan et al.
(2007) as plantas jovens de 'Paluma' foram expostas a concentrações altas e contínuas, enquanto no presente estudo as concentrações foram mais baixas e descontínuas. A ocorrência de períodos com concentrações nulas ou baixas de ozônio possibilita a recuperação da planta, reduzindo a progressão das injúrias e a correlação entre o IIF e a AOT40. Além disso, a AOT40 mede a concentração acumulada de $\mathrm{O}_{3}$ no ar, que não necessariamente é a mesma que atinge as células, uma vez que a condutância estomática é reduzida nos períodos mais secos.

Assim como P. guajava 'Paluma', muitas espécies reagem à exposição de $\mathrm{O}_{3}$ acumulando pigmentos fenólicos nas folhas, que adquirem tonalidades marrons ou avermelhadas (Sanz et al. 2002, Alves 2006). Furlan (2004) verificou um aumento na quantidade de substâncias fenólicas foliares em mudas de P. guajava 'Paluma' após fumigação com $\mathrm{O}_{3}$. Possivelmente o pigmento avermelhado acumulado nos vacúolos em folhas de Paluma seja antocianina, cuja produção é associada a estresses ambientais como seca, excesso de luz, radiação UV-B, metais pesados, e que, inclusive, pode atuar como antioxidante, neutralizando espécies ativas de oxigênio (Gould 2004). Nesse tipo de sintoma visível o pigmento fenólico fica acumulado no vacúolo e não ocorre morte celular.

Pina \& Moraes (2007) realizaram estudo em condições de campo com esta mesma cultivar, mas com várias exposições e verificaram que o tempo e a concentração acumulada de $\mathrm{O}_{3}$ quando do início da manifestação de danos variaram entre 7 e 40 dias e 174 e $590 \mathrm{ppb}$ h. Neste estudo, as injúrias iniciaram após 30 dias e 556 ppb h de $\mathrm{O}_{3}$. Esta variação ocorre porque as condições climáticas como temperatura, 
umidade relativa, radiação e velocidade dos ventos afetam a condutância estomática e conseqüentemente, a concentração de $\mathrm{O}_{3}$ absorvida pela planta e a expressão de injúrias (Mills 2003, Novak et al. 2003). Além disso, a variabilidade genética intra-específica também determina respostas diferentes (Chappelka et al. 2003).
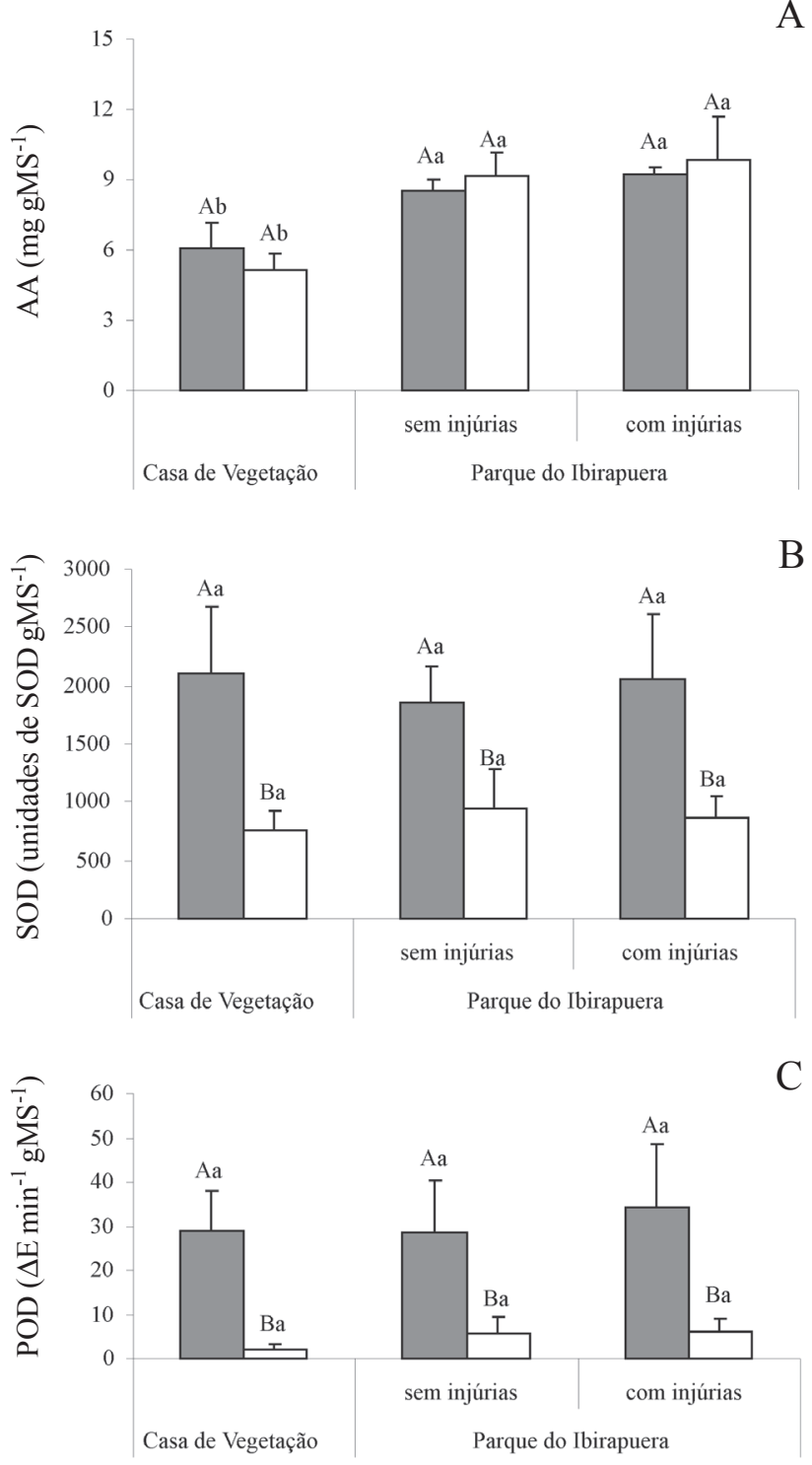

Figura 1. Antioxidantes em folhas novas e velhas, com e sem injúrias, de plantas de P. guajava 'Paluma' mantidas em casa de vegetação com ar filtrado e no Parque Municipal do Ibirapuera (SP), $\mathrm{n}=6 . \square=$ Folhas novas, $\square=$ Folhas velhas. A. concentração média de ácido ascórbico (AA); B. atividade da superóxido dismutase (SOD); C. atividade da peroxidase (POD). Barras indicam o desvio-padrão da média; letras minúsculas comparam folhas de idades iguais em ambientes diferentes e letras maiúsculas comparam folhas de idades diferentes em um mesmo ambiente $(p<0,05)$.
Tabela 3. Massa seca de folhas, caules e raízes ( $g$, média \pm desvio padrão, $\mathrm{n}=13$ ) em plantas jovens de Psidium guajava 'Paluma' mantidas em casa de vegetação com ar filtrado e no Parque Municipal do Ibirapuera (SP). Letras diferentes indicam diferenças significativas $(\mathrm{p}<0,05)$

\begin{tabular}{ccc}
\hline Massa seca $(\mathrm{g})$ & Casa de Vegetação & Ibirapuera \\
\hline Folhas & $6,9 \pm 2,5 \mathrm{a}$ & $5,9 \pm 1,0 \mathrm{a}$ \\
Caules & $4,6 \pm 1,9 \mathrm{a}$ & $4,3 \pm 0,8 \mathrm{a}$ \\
Raízes & $5,9 \pm 2,2 \mathrm{a}$ & $6,1 \pm 1,0 \mathrm{a}$ \\
\hline
\end{tabular}

Dentre os antioxidantes avaliados, apenas o ácido ascórbico apresentou resposta que pode ser atribuída à ação do $\mathrm{O}_{3}$, com o aumento de sua concentração nas plantas mantidas na área poluída. Bulbovas (2005) verificou que folhas de Caesalpinia echinata expostas na mesma região da cidade de São Paulo apresentaram maior concentração de AA do que as mantidas sob ar filtrado. Burkey et al. (2006), estudando três espécies nativas americanas, verificaram que a sensibilidade ao $\mathrm{O}_{3}$ é afetada pelo conteúdo e capacidade de regeneração do ácido ascórbico, com as espécies que apresentavam concentração basal mais alta de AA sendo as menos sensíveis ao $\mathrm{O}_{3}$. $\mathrm{O}$ aumento da concentração de AA em folhas de $P$. guajava 'Paluma' que foram mantidas no ambiente poluído e não apresentavam sintomas visíveis é considerada uma injúria latente, pois indica que ocorreram alterações em plantas que aparentemente não apresentavam evidências de estresse.

A atividade das enzimas SOD e POD diferiu apenas com relação à idade da folha, possivelmente refletindo o envelhecimento natural da mesma, uma vez que o aumento da atividade dessas enzimas foi verificado também nas plantas que permaneceram sob ar filtrado. Portanto, a atividade destas duas enzimas, não foi suficiente para conferir proteção contra a manifestação de injúrias foliares visíveis nessa espécie.

É possível que nessa espécie a proteção contra o estresse oxidativo seja feita principalmente por flavonóides, como a antocianina, ou que a síntese destes constitua a principal linha de defesa. $O$ fato de não ter ocorrido alterações no acúmulo de biomassa nas plantas expostas ao estresse, reforça a idéia de que essa pigmentação atue como uma forma de proteção e não envolvendo morte celular, embora em longo prazo o investimento na síntese de antocianinas pode ter algum custo para a planta, pois parte da 
energia e produtos fotoassimilados que seriam direcionados ao crescimento são empregados nesse tipo de proteção.

Novos estudos devem ser realizados para verificar se a atividade das enzimas SOD e POD não foi alterada devido às concentrações de $\mathrm{O}_{3}$ não terem sido muito altas ou se mesmo quando isso ocorre, os flavonóides é que desempenham o papel principal na defesa desta espécie. Outra questão a elucidar é a discriminação da importância relativa dos diversos fatores ambientais estressantes a que as plantas são expostas em experimentos de campo em locais como o Ibirapuera, os quais incluem, além das variáveis climáticas, a ocorrência freqüente de concentrações fitotóxicas de ozônio.

\section{Agradecimentos}

As autoras agradecem ao Programa Institucional de Bolsas de Iniciação Científica - PIBIC-CNPq pela bolsa concedida a Ana Paula de Souza Dias.

\section{Literatura citada}

Alves, V. 2006. Avaliação do potencial bioindicador de Psidium cattleyanum Sabine e P. guajava L. cv. Paluma à poluição atmosférica na cidade de São Paulo. Tese de doutorado, Universidade de São Paulo, São Paulo.

Arndt, U. \& Schweizer, B. 1991. The use of bioindicators for environmental monitoring in tropical and subtropical countries. In: H. Ellenberg, U. Arndt, B. Bretthauer, B. Ruthsatz \& L. Steubing (eds.). Biological Monitoring: signals from the environment. Vieweg \& Sons, Eschborn, pp. 199-260.

Bowler, C., Montagu, M.V. \& Inzé, D. 1992. Superoxide dismutase and stress tolerance. Annual Review of Plant Physiology and Plant Molecular Biology 43: 83-116.

Bray, E.A., Baylei-Senes, J. \& Weretylni, K.E. 2000. Responses to abiotic stress. In: B.B. Buchanan, W. Gruissen \& R.L. Jones (eds.). Biochemistry and Molecular Biology of Plants. American Society of Plant Physiologists, New York, pp. 1158-1203.

Bulbovas, P. 2005. Defesas antioxidativas em plantas jovens de Caesalpinia echinata Lam. (pau-brasil) como indicadoras de resistência da espécie à poluição atmosférica na cidade de São Paulo, SP. Tese de doutorado, Universidade de São Paulo, São Paulo.

Burkey, K.O., Neufeld, H.S., Souza, L., Chappelka, A.H. \& Davison, A.W. 2006. Seasonal profiles of leaf ascorbic acid content and redox state in ozone-sensitive wildflowers. Environmental Pollution 143: 427-434.
CETESB. 2006. Companhia de Tecnologia de Saneamento Ambiental. 2006. Qualidade do ar no Estado de São Paulo: série relatórios 2005. CETESB, São Paulo.

Chameides, W. 1989. The chemistry of ozone deposition to plant leaves: the role of ascorbic acid. Environmental Science and Technology 23: 595-600.

Chappelka, A., Renfro, J., Somers, G. \& Nash, B. 1997. Evaluation of ozone injury on foliage of blackcherry (Prunus serotina) and tall milkweed (Asclepias exalata) in Great Smoky Mountains National Park. Environmental Pollution 95: 3-18.

Chappelka, A.H., Neufeld, H.S., Davison, A.W., Somers, G.L. \& Renfro, J.R. 2003. Ozone injury on cutleaf coneflower (Rudbeckia laciniata) and crown-beard (Verbesina occidentalis) in Great Smoky Mountains National Park. Environmental Pollution 125: 53-59.

Dassler, H.G. \& Bortitz, S. 1988. Air pollution and its influence on vegetation. Jung, Dordrecht. In: B.B. Buchanan, W. Gruissen \& R.L. Jones (eds.). Biochemistry and Molecular Biology of Plants. American Society of Plant Physiologists, New York, pp. 1158-1203.

De Temmerman, L., Bell, J.N.B., Garrec, J.P., Klumpp, A., Krause, G.H.M. \& Tonneijck, A.E.G. 2004. Biomonitoring of air pollutants with plants considerations for the future. In: A. Klumpp, W. Ansel \& G. Klumpp (eds.). Urban Air Pollution, Bioindication and Environmental Awareness. Cuvillier Verlag, Göttingen, pp. 337-374.

Filella, I., Peñuelas, J. \& Ribas, A. 2005. Using plants biomonitors and flux modelling to develop ozoneresponse realtionships in Catalonia. Environmental Pollution 134: 145-151.

Fuhrer, J., Skarby, L. \& Ashmore, M.R. 1997. Critical levels for ozone effects on vegetation in Europe. Environmental Pollution 97: 91-106.

Furlan, C.M. 2004. Efeitos de poluentes atmosféricos na composição química de indivíduos jovens de Tibouchina pulchra (Cham.) Cogn. e Psidium guajava L.. Tese de doutorado, Universidade de São Paulo, São Paulo.

Furlan, C.M., Moraes, R.M., Bulbovas, P., Domingos, M., Salatino, A. \& Sanz, M.J. 2007. Psidium guajava 'Paluma' as a new bioindicator of ozone in the tropics. Environmental Pollution 147: 691-695.

Gadallah, M.A.A. 2000. Effects of acid mist and ascorbic acid treatment on the growth, stability of leaf membranes, chlorophyll content and some mineral elements of Carthamus tinctorius, the safflower. Water, Air, and Soil Pollution 118: 311-327.

Gould, K.S. 2004. Nature's swiss army knife: the diverse protective roles of anthocyanins in leaves. Journal of Biomedicine and Biotechnology 5: 314-320. 
ICP-forests. 2006. Submanual for the assessment of ozone injurynon European forest ecosystems. www.gva.es/ ceam/ICP-forests. (acesso em 30.10.2006).

Iqbal, M., Abdin, M.Z., Mahmooduzzafar, Yunus, M. \& Agrawal, M. 1996. Resistance mechanism in plants against air pollution. In: M. Yunus \& M. Iqbal (eds.). Plant Response to Air Pollution. John Wiley and Sons, Chichester, pp. 195-240.

Keller, T. \& Schweizer, H. 1977. Air pollution and ascorbic acid. European Journal of Forest Pathology 7: 338-350.

Klumpp, A., Ansel, W., Klumpp, G., Vergne, P., Sifakis, N., Sanz, M.J., Rasmussen, S., Ro-Poulsen, H., Ribas, A., Peñuelas, J., Kambezidis, H., He, S., Garrec, J.P. \& Calatayud, V. 2006. Ozone pollution and ozone biomonitoring in European cities. Part II. Ozone-induced plant injury and its relationship with descriptors of ozone pollution. Atmospheric Environment 40: 7437-7448.

Klumpp, G., Guderian, R. \& Küppers, K. 1989. Peroxidaseund superoxiddismutase- aktivität sowie prolingehalte von fichtennadeln nach belastung mit $\mathrm{O}_{3}, \mathrm{SO}_{2}$ und $\mathrm{NO}_{2}$. European Journal of Forest Pathology 19: 84-97.

Krupa, S., McGrath, M.T., Andersen, C.P., Booker, F.L., Burkey, K.O., Chappelka, A.H., Chevone, B.I., Pell, E.J. \& Zilinskas, B.A. 2001. Ambient ozone and plant health. Plant Disease 85: 4-12.
Manning, W.J. 2003. Detecting plants effects is necessary to give biological significance to ambient ozone monitoring data and predictive ozone standards. Environmental Pollution 126: 375-379.

Mills, G. 2003. Modification of plant response by environment conditions. In: J.N.B. Bell \& M. Treshow (eds.). Air pollution and plant life. $2^{\text {nd }}$ ed., John Wiley and Sons, Chichester, pp. 343-358.

Novak, K., Skelly, J.M., Schaub, M., Krauchi, N., Hug, C., Werner, L. \& Bleuler, P. 2003. Ozone air pollution and foliar injury development on native plants of Switzerland. Environmetal Pollution 125: 41-52.

Osswald, W.F., Kraus, R., Hippeli, S., Benz, B., Volpert, R. \& Elstner, E.F. 1992. Comparison of the enzimatic actives of dehydroascorbic acid redutase, glutathione redutase, catalase, peroxidse and superoxide dismutase of healthy and damaged spruce needles (Picea abies (L.) Karst). Plant Physiology 139: 742-748.

Pell, E.J., Eckardt, N.A. \& Glick, R.E. 1994. Biochemical and molecular basis for impairment of photosynthesis potential. Photosynthesis Research 39: 453-462.

Pina, J.M. \& Moraes, R.M. 2007. Ozone-induced foliar injury in saplings of Psidium guajava 'Paluma' in São Paulo, Brazil. Chemosphere 66: 1310-1314.

Sanz, M.J., Sanchez, G., Calatayud, V., Gallego, M.T. \& Cervero, J. 2002. La contaminación atmosférica de los bosques: Guia para la indentificacion de los daños visibles causados por ozone. Ministerio Del Medio Ambiente, Madrid. 